\title{
Reversible Optical Data Storage on Poly(Ethylene Terephthalate)
}

\author{
G. S. BUCKLEY* and C. M. ROLAND \\ Chemistry Division, Code 6120 \\ Naval Research Laboratory \\ Washington D.C. 20375-5342
}

\begin{abstract}
A desirable feature of optical data storage processes is that they provide high contrast between the image and background. Thermal marking techniques are inherently advantageous in this regard, because of the nonlinearity of the response. A thermal method for lithography on polymer films has been developed based on selective exposure of the films to infrared laser radiation passing through a mask. Radiation induced crystallization, melting and ablation have been demonstrated with the processes prevailing at different levels of radiation intensity. These methods are capable of producing high resolution images with excellent edge acuity and minimal interference from diffraction. The absence of diffraction effects is due to the nonlinear response of the polymer film to the radiation. The best resolution obtained to date (submicron) is limited by the size of the smallest features present on the masks used to create the pattern. The ultimate resolution achievable by these methods is presently unknown.
\end{abstract}

\section{INTRODUCTION}

T There is considerable interest in the use of polymers as media for optical data storage, with a variety of techniques employed, such as chemical deposition, doping, ablation, etc. (1-8). Commercial feasibility of any microlithographic method requires that various criteria be met, including marking speed and sensitivity ( $\sim 1 \mathrm{~nJ}$ per mark or lower), and the production of images which are acute, indelible, and durable $(>30$ year lifetime for archiving).

A potential material for the storage of submicron optical images is poly(ethylene terephthalate) (PET), a low-cost plastic with good mechanical properties. Rapid, nonequilibrium heating can induce chemical bond rupture in PET; removal by vaporization of the decomposition by-products creates a three-dimensional image. Using an ultraviolet laser, such ablation has been used to produce diffraction gratings in PET with periodicities as small as $0.24 \mu \mathrm{m}$ (9). Ablation of semicrystalline PET with infrared laser radiation has also been carried out; however, only very coarse, illdefined images were produced, because of interference from the crystallites (10). More recently it was shown (11-13) that intricate, high-quality images, smaller than $1 \mu \mathrm{m}$, could be produced by ablation of PET with $\mathrm{CO}_{2}$ laser radiation $(10.6 \mu \mathrm{m})$ passed through a mask. This ablation is regarded as a ther-

- ASEE visiting fellow, permanent address: Cameron University, Lawton, OK mal process because a single infrared photon provides insufficient energy to effect the marking event. Thermal processes are inherently nonlinear, since the extent of the medium's response (i.e., the development of an ablated pit) is not simply proportional to the input intensity. Such nonlinearity provides for the inherently good contrast and resolution of such a technique. There is no diffraction limitation on the resolution, although the latter will ultimately be limited by thermal diffusion away from the irradiated region.

It has been found $(12,13)$ that infrared irradiation with powers lower than that used for ablation will effect crystallization of initially amorphous PET. Similar to the ablation process, images smaller than the $10.6 \mu \mathrm{m}$ wavelength can be produced. Unlike the more common technique of crystallizing small molecule species residing within a polymeric matrix (14-18), in this case the polymer serves both as the active medium and the substrate. This simplifying of the processing can be a commercial advantage. Unfortunately, very few polymers are candidates for microlithography via crystallization. The material must be initially amorphous, yet highly crystallizable. Few polymers crystallize at rates sufficiently slow that they can be obtained in an initial amorphous state. In addition to PET, others meeting this criteria include poly(phenylene sulfide) (19) and poly(ether ether ketone) (20). However, when crystallized with an infrared laser, the images produced on these materials are substantially larger than what can be achieved with 
PET. The resolution is limited by diffusion of the heat away from the directly irradiated regions. It is unclear why PET is not similarly affected by thermal smearing (13); it appears to be unique in this regard.

An obvious advantage of using a physical change such as crystallization as the basis for lithography is the reversibility of the process, enabling multiple write / erase cycles. In fact, erasure via melting of crystalline images by reapplication of the laser radiation has been demonstrated with PET $(12,13)$. The crystalline marking can be reproduced, without apparent degradation of the image quality, by re-exposure to an appropriate level of the infrared radiation. Thus, writing and erasing can be successively executed by simple adjustment in the intensity or duration of the irradiation. A disordering process such as melting can be accomplished significantly faster than the reverse operation of ordering the polymer segments into a crystalline phase; therefore, it is anticipated that induced melting of a crystalline film is potentially faster than a lithographic process based on crystallization of initially amorphous material. The objective of the present study was to explore this issue, and attempt to systematically define the range of variables over which PET can be crystallized or melted via laser irradiation.

\section{EXPERIMENTAL}

The PET (Eastman Chemicals, Kingsport, TN) had an intrinsic viscosity of $0.75 \mathrm{dL} / \mathrm{g}$. Where desired, the initially amorphous film $(0.13 \mathrm{~mm}$ thick) was crystallized by heating in a Carver Press at $20^{\circ} \mathrm{C} / \mathrm{min}$ from $25^{\circ} \mathrm{C}$ to $175^{\circ} \mathrm{C}$. This yields a $20 \%$ degree of crystallinity as determined by differential scanning calorimetry (Perkin Elmer Model 7).

A California Model LS-55-ATVO cw laser was used to irradiate the film. The laser wavelength was tunable between $930 \mathrm{~cm}^{-1}$ and $1090 \mathrm{~cm}^{-1}$, with a maximum power of $6.3 \mathrm{~W}$. The intensity was measured with a Coherent General Thermal Sensor. All values reported herein refer to the on-axis intensity (i.e., the peak of the Gaussian laser beam profile), which is a factor of $33 \%$ higher than the intensity averaged over the beam waist. This beam waist, defined as the radial distance from the beam center at which the electric field intensity is down by $1 / \mathrm{e}$, was determined to be $0.7 \mathrm{~mm}$ at the center of the laser tube. It diverges with the square of the distance from the laser.

Masks, consisting of GaAs with gold plating, were fabricated at the NanoElectronic Fabrication Facility of the Naval Research Laboratory. Short time exposure of the PET to the light was achieved using a Stanford Research Systems Model SR 570 chopper, with apertures of varying size. This allowed irradiation times as brief as $0.03 \mathrm{~s}$. Irradiation for more extended duration (e.g., several seconds) was carried out without the chopper. Infrared spectra of the films were collected with a Nicolet Magna-IR 750 Fourier Transform Infrared spectrophotometer.

\section{RESULTS}

\section{Amorphous to Cryetalline Phase Transition}

The absorbance of the PET, as well as the intensity and duration of the irradiation, govern the crystallization response of the film. Three wavelengths were used herein, $935 \mathrm{~cm}^{-1}, 976 \mathrm{~cm}^{-1}$, and $1079 \mathrm{~cm}^{-1}$, corresponding to absorption coefficients of $12 \mathrm{~mm}^{-1}, 22$ $\mathrm{mm}^{-1}$ and $>50 \mathrm{~mm}^{-1}$, respectively. For the $0.13 \mathrm{~mm}$ films in their initial amorphous state, the respective transmissions at these wavelengths were $22 \%, 6 \%$, and $0 \%$. The effect of irradiation was determined by sampling the film after different exposure times, and evaluating the extent of crystallinity using DSC. Typical results are shown in Fig. 1. As expected, crystallinity transpires at a faster rate for the more strongly absorbing wavelength $\left(1079 \mathrm{~cm}^{-1}\right)$. The ultimate degree of crystallinity, after sufficient irradiation, is the same for the three laser wavelengths. Surprisingly, although the absorbance for different wavelengths varies greatly, the image quality did not significantly differ. This suggests that thermal equilibrium is established and the clarity of the images is not a consequence of nonequilibrium conditions $(12,13)$.

Typical crystalline images induced in amorphous PET by radiation heating through a mask are shown in Fig. 2. In principle, images smaller than the wavelength of the radiation can be obtained because of the nonlinear response of a thermal process, whereby diffraction limitations are circumvented. Nevertheless, diffracted radiation still reaches the film, giving rise to a high level of "noise," or spurious crystallization, as seen in Fig. 2. Some improvement of image quality is achievable with the use of a heat sink, such as metal backing placed on the film. However, some spurious crystallization, presumably from diffracted light, is always in evidence.

Although the two variables, intensity and exposure time, might be expected to correlate, too high an in-

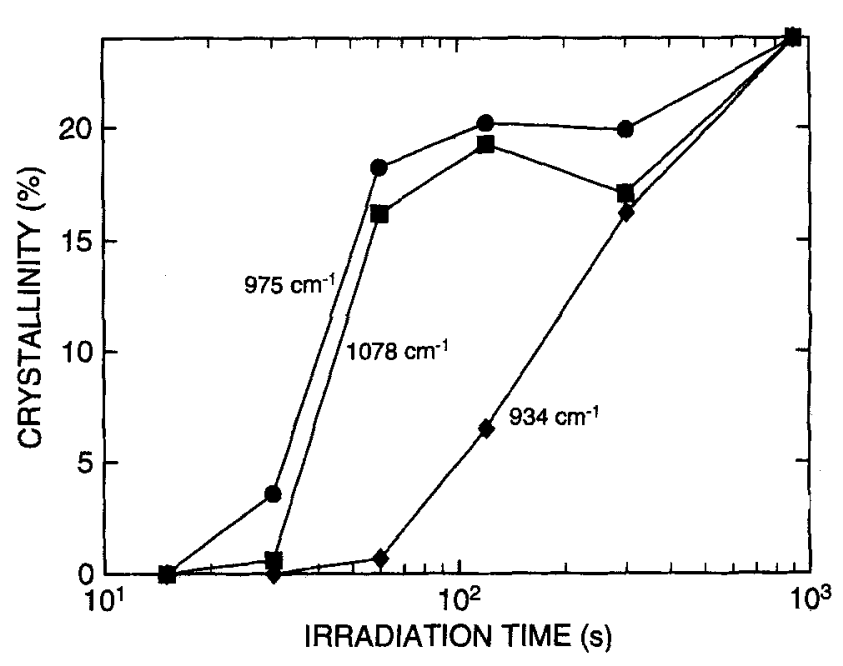

Fig. 1. Degree of crystallinity obtained in PET film as a function of exposure time, using infrared radiation of the indicated frequency. 


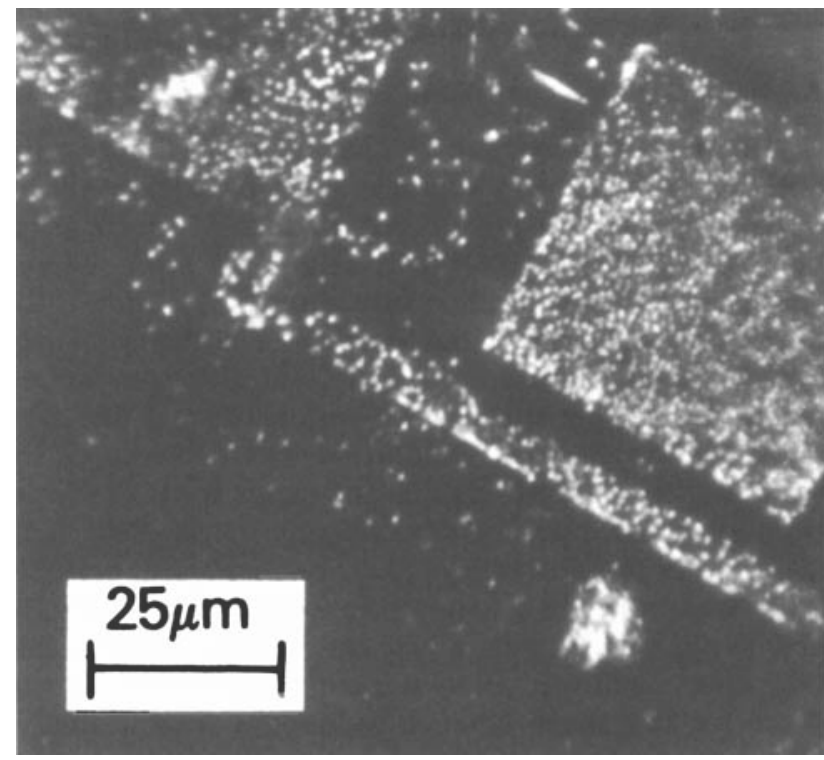

Fig. 2. Amorphous PET film after irradiation through a light field gold on GaAs mask.

tensity raises the local film temperature sufficiently high to preclude crystallization. For example, regardless of the duration of the irradiation, when the film was exposed to the beam at intensities above a few watts per $\mathrm{cm}^{2}$, a clear central spot formed, often encompassed by a circular crystalline fringe. At higher powers, a hole develops almost instantly in the center. No crystallinity was ever detectable in the central region; evidently it was heated too high, too fast for the PET to crystallize. The weakly crystalline fringe is believed to be the result of thermal diffusion away from the directly irradiated central spot. This fringe only appeared after irradiation for at least $15 \mathrm{~s}$ with on-axis intensities exceeding $2 \mathrm{~W} / \mathrm{cm}^{2}$.

The range of conditions under which we have successfully crystallized PET is narrow -1.7 to $2.3 \mathrm{~W} / \mathrm{cm}^{2}$ on-axis intensity and 30 to 120 s exposure time-and too slow for commercial applications. However, one can crystallize PET in shorter times using conductive heating, which suggests that radiation-induced crystallization under the proper, specific conditions could be accomplished in times shorter than achieved herein. While requiring higher laser power, this would also minimize thermal diffusion, enabling higher resolution. The difficulty is that an increase in intensity above that required for crystallization can result in the ablation of the amorphous PET, rather than faster crystallization. Note that the power requirements for ablation are not substantially greater than those required for crystallization-intensities ranging from 3.0 to $12 \mathrm{~W} / \mathrm{cm}^{2}$, with exposure times from 30 to $120 \mathrm{~s}$. The images obtained by ablation (Fig. 3) are very sharp, having higher optical quality than the crystalline images. The response of the PET to the ablation process is much more nonlinear than crystallization or melting $(11,12)$; consequently, the diffracted radiation provides insufficient energy to etch the film surface in the manner of the primary beam.

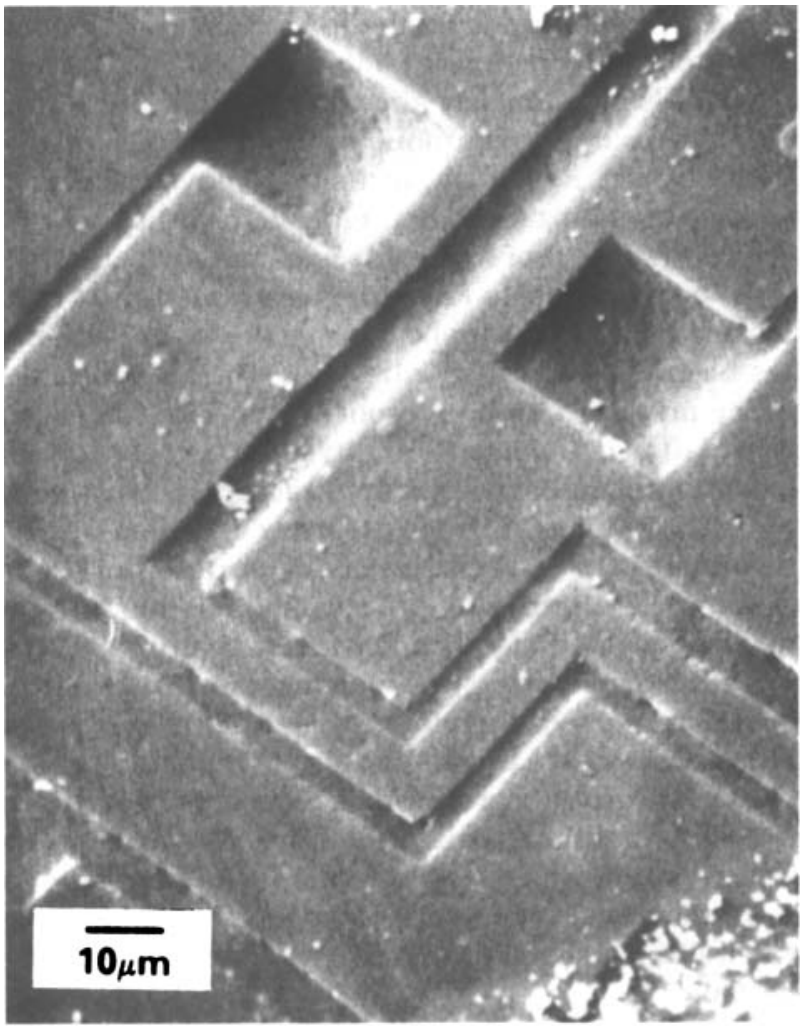

Fig. 3. Same as Fig. 2, but at higher radiation intensities, giving rise to ablation of the PET.

Interestingly, formation of ablated images in the irradiated region of the film occurred sporadically. We believe this is attributable to the varying spatial quality of the film (e.g., thickness), along with nonuniformity of the laser beam profile. Since the mask is in direct contact with the top (irradiated) side of the film. it probably functions as a heat sink. While this minimizes thermal diffusion and thereby contributes to image quality, waviness in the film will result in inconsistent contact, potentially introducing variability into the process. This aspect of PET ablation requires further study.

\section{Melting of Crystalline PET}

The melting of semi-crystalline PET offers a faster, and potentially higher resolution, alternative lithographic method. The process should proceed much faster than crystallization, be operative over a broader range of temperatures, and thermal diffusion effects might be minimized to some degree given the endothermic nature of melting. To melt PET requires higher intensity and/or shorter duration irradiation. Radiation intensities - actually incident on the film surface - as high as $46 \mathrm{w} / \mathrm{cm}^{2}$ induced melting of initially crystalline PET in times as short as $0.12 \mathrm{~s}$ (see Fig. 4). This value refers to the on-axis intensity; the average local intensity was lower. Beyond this power level, hole formation and ablation transpired. At the other extreme, on-axis intensities as low as $3.6 \mathrm{w} / \mathrm{cm}^{2}$ were sufficient to melt the PET, although 20 to $30 \mathrm{~s}$ 


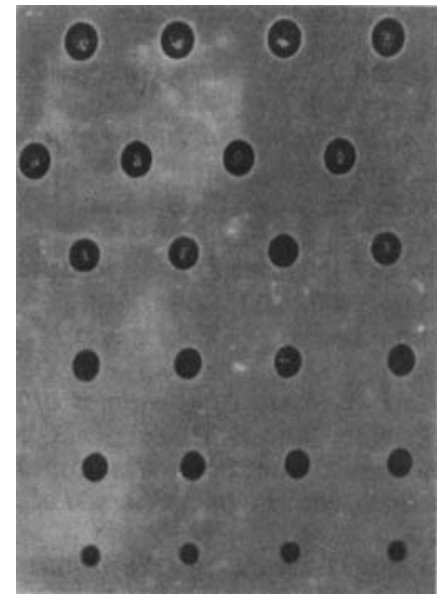

Fig. 4. Illustrative results for laser-induced melting of a crystalline PET film, with an on-axis intensity of $47 \mathrm{~W} / \mathrm{cm}^{2}$ (the average intensity is lower). No patterning mask was used, so that the images reflect the incident laser beam. The rows correspond to irradiation times of $0.15 \mathrm{~s}, 0.21 \mathrm{~s}, 0.25 \mathrm{~s}$, $0.28 \mathrm{~s}, 0.35 \mathrm{~s}$, and $0.42 \mathrm{~s}$, increasing from bottom to top; columns represent repeat runs using identical conditions. Note that at the longer times, some bubbling of the film is evident.

were required. At still lower powers, no discernible change in the films was observed, other than slight warming. In Fig. 5 we summarize the intensity-time relationships determined experimentally for the three processes-crystallization, ablation, and melting. While short time regimes are best for resolution, since thermal diffusion effects would be minimized, the former are limited to melting with the experimental variables available in the present study.

Attempts to employ the GaAs masks in the production of images via melting suffered from the same poor reproducibility found for ablation. While images could be obtained by irradiating for $0.1 \mathrm{~s}$ or longer at intensities in the range from 3 to $21 \mathrm{w} / \mathrm{cm}^{2}$, they were of generally poor quality.

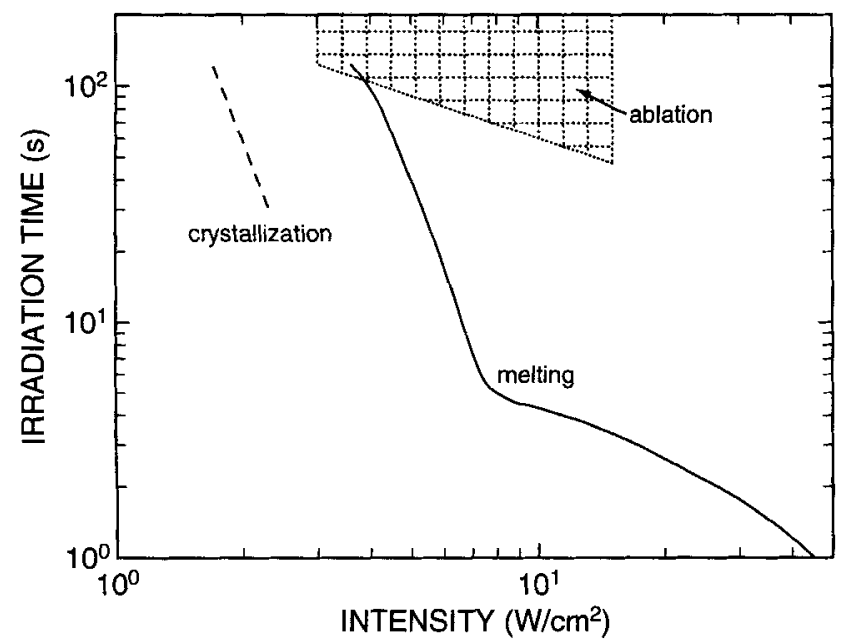

Fig. 5. Comparison of conditions of on-axis radiation intensity and duration necessary to achieve marking of the PET film by means of the indicated mechanisms. Note that extended exposure to intense radiation causes hole formation in the film.
In order to eliminate the mask as a variable, a series of experiments were conducted by passing the laser beam through a $150 \mu \mathrm{m}$ slit, whereby the diffracted beam serves as the illuminating source. Figure 6 shows representative results of the image formed in the initially crystalline, opaque PET film. Melting transpires to create a sharp, transparent line, about 25 $\mu \mathrm{m}$ wide. The primary beam intensity produces a melted image that faithfully reproduces the beam dimensions. However, this process is accompanied by the formation of translucent areas adjacent to the melted area, spaced laterally from the central line by roughly $190 \mu \mathrm{m}$. These diffuse bands represent partially melted PET, caused by radiation diffracted from the main beam (21). As the process proceeds, such satellite images become larger, eventually coalescing with the central region to produce a completely transparent ellipse. This demonstrates that while diffraction limitations can be circumvented because of the nonlinear nature of the material's response, high resolution imaging with long wavelength radiation will always entail the potential for "noise" and smearing of the images. Avoiding these interferences requires careful control of the intensity and duration of the irradiation.

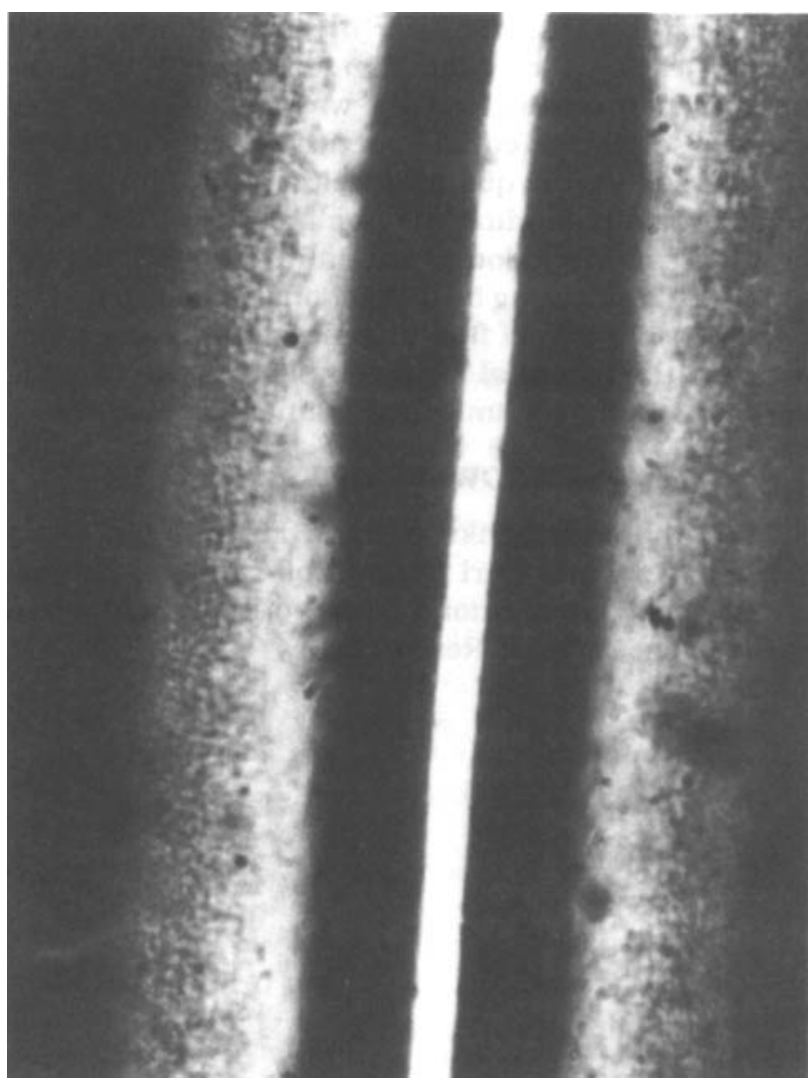

Fig. 6. Image formed in an initially crystalline, opaque film after irradiation with the laser beam passed through a 150 $\mu \mathrm{m}$ slit. The central white line, $25 \mu \mathrm{m}$ wide, represents a transparent, amorphous section, which is flanked by diffuse, translucent bands, arising from diffracted intensity. 


\section{CONCLUSIONS}

Crystallization of PET produces surprisingly small images. The localized crystallization in PET is much less affected by thermal diffusion than is the case for similarly slow-crystallizing polymers. However, for commercial application, the radiation-induced crystallization is too slow. Although increases in the crystallization rate can be realized through orientation or the addition of nucleating agents, any gains would likely be offset by reductions in the obtainable resolution. Simply increasing the radiation intensity does not accelerate the process. Instead, crystallization does not occur at all. Local temperatures exceeding the crystalline melting point $\left(\sim 250^{\circ} \mathrm{C}\right)$ are reached, with only flow or bubbling of the molten film resulting. At even higher intensity levels, ablation transpires; this process yields the highest image quality, although, of course, it is not reversible.

There is a range of radiation intensity for which the temperature of the PET is rapidly taken above the melting point, while remaining low enough that flow or ablation is avoided. In this regime microlithography can be accomplished by melting of initially crystalline PET. This process is much faster than laser-induced crystallization, with time scales as short as $\sim 0.1 \mathrm{~s}$ achieved herein. It also retains the advantage of being reversible. The transparent images could be routinely "erased" by re-crystallization of the PET, accomplished either with the laser (at lower power) or by simply heating the entire film to above $120^{\circ} \mathrm{C}$. Although no extensive testing was carried out, this write-erase process could be repeated without apparent degradation in quality of either the film or the image. Notwithstanding the nonlinearity of the melting process, diffraction of the infrared light was observed. This smearing by diffraction can be avoided by judicious selection of film thickness and laser power; however, the potential for it complicates the imaging and may ultimately limit the obtainable resolution.

\section{ACKNOWLEDGMENTS}

The authors would like to thank Jason Green, Carla Bartholomew, and Carl Bennett of Cameron University for their contributions. This work was supported by the Office of Naval Research.

\section{REFERENCES}

1. D. J. Ehrlich and J. Y. Tsao, J. Vac. Sci. Technol., B 1(4), 969 (1983).

2. The Effects of Radiation on High-Technology Polymers, Vol. 381, E. Reichmanis and J. O'Donnell, eds., ACS Symposium Series; Amer. Chem. Soc.; Washington, D.C. (1989).

3. "Symposium on Polymers in Microlithography," Poly. Mater. Sci. Eng., 60, 40 (1989).

4. E. Reichmanis and L. F. Thompson, Chem. Rev., 88, 1273 (1989).

5. Electronic and Photonic Applications of Polymers, Vol. 218, M. J. Bowden and S. R. Turner, eds., Advances in Chemistry; Amer. Chem. Soc.: Washington, D.C. (1988).

6. Am. Chem. Soc., Div. Polym. Chem. Polym. Prepr., 29. 195 (1988).

7. Polymers for High Technology - Electronics and Photonics, Vol. 346. M. J. Bowden and S. R. Turner, eds., ACS Symposium Series; Amer. Chem. Soc.; Washington, D.C. (1987).

8. Radiation Effects on Polymers, ACS Symposium Sertes 475, R. L. Clough and S. W. Shalaby, eds. (1991).

9. K. J. Ilcisin and R. Fedosejevs, Appled Optics, 26, 396 (1987).

10. J. E. Andrew, P. E. Dyer, D. Forster, and P. H. Key, Appt. Phys. Lett., 43, 717 (1983).

11. M. F. Sonnenschein and C. M. Roland, Appl. Phys. Lett., 57, 425 (1990).

12. "Thermal Marking of Amorphous Poly(Ethylene Terephthaiate)," C. M. Roland, J. P. Armistead, and M. F. Sonnenschein, in ref. 8, Chapter 21.

13. M. F. Sonnenschein, A. M. Kotliar, and C. M. Roland, Polym. Eng. Sci., 30, 1165 (1990).

14. A. Werner, H. Hibst, and J. Petermann, U.S. Patent 4,766,021 (1988).

15. M. Takenaga, N. Yamada, K. Nishiuchi, N. Akihara, T. Ohta, S. Nakamura and Y. Yamashita, J. Appl. Phys., 54, 5376 (1983).

16. K. Ota, Japanese Patent \#58,199,345A (1984).

17. V. Novotny and L. Alexandru, J. Appl. Polym. Sci., 24, 1321 (1979).

18. S. R. Ovshinsky and H. Fritzsche, IEEE Trans. Elec. Dev., ED-20, 91 (1973).

19. M. F. Rubner and P. Cukor, U.S. Patent $4,486,463$ (1984).

20. S.-J. Fang, R. Salovey, and S. D. Allen, Polym. Eng. Sci., 29, 1241 (1989).

21. J. A. Murphy and A. Egan, Eur. J. Phys., 14, 121 (1993).

Reviston received June 25, 1996 